\title{
CANONICAL THEORY OF THE NONPARAMETRIC LAGRANGIAN MULTIPLE INTEGRAL PROBLEMS WITH VARIABLE BOUNDARIES ${ }^{1}$
}

\author{
BY HANS RUD. WEBER
}

\author{
Communicated by C. B. Morrey, Jr., October 21, 1968
}

1. Introduction. The aims of this work were

(1) to establish a canonical theory of the nonparametric multiple integral problems with variable boundaries which is simpler than the Carathéodory theory [1] and contains fewer variables;

(2) to choose the canonical variables so that by specializing to simple integrals the theory reduces to the equations which are known from Carathéodory's textbook [2], and

(3) to suppose from the beginning that the problem is of the Lagrange type, i.e. that there is a set of differential equations which the extremals must satisfy.

The results obtained are stated in the following sections.

2. The problem. Our problem is to minimize the integral

$$
\int \cdots \int F\left(t^{\alpha}, x^{i}, x^{i \alpha}\right) \prod_{k=1}^{m} d t^{k} \quad(i=1,2, \cdots, n, \alpha=1,2, \cdots, m)
$$

taking into account the side conditions

$$
G_{\rho^{\prime}}\left(t^{\alpha}, x^{i}, x^{i \alpha}\right)-\Gamma_{\rho^{\prime}}=0 \quad\left(\rho^{\prime}=1,2, \cdots, p, \quad p<n m\right) .
$$

So, we number

(1) by greek indexes the independent variables,

(2) by latin indexes the dependent variables, and

(3) by greek indexes with accents the side conditions.

To speak more exactly, (2.1), (2.2) is a family of variational problems with the parameters $\Gamma_{\rho^{\prime}}$. Suppose that originally the side conditions

$$
G_{\rho^{\prime}}\left(t^{\alpha}, x^{i}, x^{i \alpha}\right)=0
$$

are given. Then, it turns out to be advantageous to replace them from the beginning by the family of side conditions (2.2) thus introducing the parameters $\Gamma_{\rho^{\prime}}$ which later on are essential in the theory.

Throughout, we suppose that

$$
F>0 \text {. }
$$

${ }^{1}$ Research work sponsored by the Deutsche Forschungsgemeinschaft. 
Original variables as $x^{i \alpha}$ have superscripts, canonical variables as $y_{\alpha i}$ have subscripts. Derivatives are denoted inversely, e.g.

$$
\frac{\partial \Phi}{\partial x^{i \alpha}}=\Phi_{i \alpha}, \quad \frac{\partial \Phi}{\partial y_{\alpha i}}=\Phi^{\alpha i} .
$$

The derivative of a determinant with respect to one of its elements is denoted by using subscripts; for example,

$$
\frac{\partial\left|a_{\rho \sigma}\right|}{\partial a_{\mu \nu}}=\left|a_{\rho \sigma}\right|_{\mu \nu}
$$

For simplification, all functions are supposed to be analytic.

3. The fundamental equations. As we have introduced the parameter $\Gamma_{\rho^{\prime}}$ in the side conditions (2.3), these parameters must also appear in the equations of the $m$ families of geodesically equimembranal surfaces. Therefore, we replace Carathéodory's equations $S_{p}\left(t^{\alpha}, x^{i}\right)$ = const by

$$
S_{p}\left(t^{\alpha}, x^{i}, \Gamma_{\lambda^{\prime}}\right)=\text { const. }
$$

In a geodesic field, the fundamental equations are shown to be

$$
\begin{gathered}
F-\left|S_{\rho, \sigma}+S_{\rho, l} x^{l \sigma}\right|=0, \\
F_{i \alpha}+\mu^{\lambda^{\prime}} G_{\lambda^{\prime}, i \alpha}-\left|S_{\rho, \sigma}+S_{\rho, l} x^{l \sigma}\right|_{\alpha \alpha} S_{\kappa, i}=0 .
\end{gathered}
$$

In order to determine the Lagrangian multipliers $\mu^{\lambda^{\prime}}$, the side conditions (2.2) must be adjoined.

When the partial derivatives $S_{\alpha, \lambda}$ and $S_{\alpha, i}$ are looked upon as free variables we put

$$
S_{\alpha, \lambda}=y_{\alpha \lambda}, \quad S_{\alpha, i}=y_{\alpha i} .
$$

For abbreviation, we define the functions

$$
\Delta=\left|y_{\rho \sigma}+y_{\rho l} x^{l \sigma}\right|
$$

and

$$
\Phi\left(t^{\alpha}, x^{i}, x^{i \alpha}, \mu^{\rho^{\prime}}, y_{\alpha \lambda}, y_{\alpha i}, \Gamma_{\rho^{\prime}}\right)=F+\mu^{\lambda^{\prime}}\left(G_{\lambda^{\prime}}-\Gamma_{\lambda^{\prime}}\right)-\Delta .
$$

Then, the equations (3.2) and (2.2) which characterize a geodesic field can be written

$$
\begin{gathered}
\Phi_{i \alpha}=0, \quad \Phi_{\rho^{\prime}}=0, \\
\Phi=0 .
\end{gathered}
$$

The subscripts $i \alpha$ and $\rho^{\prime}$ mean differentiation with respect to $x^{i \alpha}$ and $\mu^{\rho^{\prime}}$; see end of $\$ 2$. 
4. The Legendre condition in the original variables. It is shown that the analogue of Carathéodory's Legendre condition can be stated as follows: Under the side conditions

$$
\Phi_{\rho^{\prime}, j \beta} \mathrm{r}^{j \beta}=0
$$

the quadratic form

$$
\Phi_{i \alpha, j \beta} \mathrm{r}^{i \alpha} \mathrm{r}^{j \beta}
$$

must be positive definite. This requirement implies the inequality

$$
\left|\begin{array}{l}
\Phi_{i \alpha, j \beta}, \Phi_{i \alpha, \sigma^{\prime}} \\
\Phi_{\rho^{\prime}, j \beta}, 0
\end{array}\right| \neq 0 .
$$

5. Introduction of the canonical variables. We contrast the original variables

$$
x^{i \alpha}, \mu^{\rho^{\prime}}
$$

with the canonical variables

$$
y_{\alpha i}, \Gamma_{\rho^{\prime}} .
$$

In order to fix relations between these two sets of quantities, we prescribe the equations

$$
\Phi_{i \alpha}=0, \quad \Phi_{\rho^{\prime}}=0
$$

which are a part of the system (3.6), (3.7) that characterizes a geodesic field. If the Legendre condition (4.1), (4.2) holds the relations (5.3) can be solved for the original variables (5.1). In a geodesic field, they can be solved for the canonical variables (5.2).

6. The analogue to the Hamilton-Jacobi equation. If Legendre's condition holds the original variables (5.1) can be eliminated from the equations (3.6), (3.7). Thus, a relation

$$
\mathrm{H}\left(t^{\alpha}, x^{i}, y_{\alpha \lambda}, y_{\alpha i}, \Gamma_{\rho^{\prime}}\right)=0
$$

is obtained, and, because of (3.3),

$$
\mathrm{H}\left(t^{\alpha}, x^{i}, \frac{\partial S_{\alpha}}{\partial t^{\lambda}}, \frac{\partial S_{\alpha}}{\partial x^{i}}, \quad \Gamma_{\rho^{\prime}}\right)=0
$$

is the analogue to the Hamilton-Jacobi partial differential equation. If $\mathfrak{S}=0$ the relations

$$
\begin{aligned}
& F_{\alpha}+\mu^{\lambda^{\prime}} G_{\lambda^{\prime}, \alpha}=-\lambda \mathrm{H}_{\alpha}, \\
& F_{i}+\mu^{\lambda^{\prime}} G_{\lambda^{\prime}, i}=-\lambda \mathrm{H}_{i},
\end{aligned}
$$




$$
\begin{aligned}
\Delta^{\alpha \lambda} & =\lambda H^{\alpha \lambda}, \\
\Delta^{\alpha \kappa} x^{i \alpha}=\Delta^{\alpha i} & =\lambda H^{\alpha i}, \\
\mu^{\rho^{\prime}} & =\lambda H^{\rho^{\prime}}
\end{aligned}
$$

hold where

$$
\lambda=\frac{m\left|y_{\alpha k} \mathrm{H}^{\lambda \kappa}+y_{\alpha i} \mathrm{H}^{\lambda i}\right|}{\left|\mathrm{H}^{\rho \sigma}\right|\left(y_{\alpha \lambda} \mathrm{H}^{\alpha \lambda}+y_{\alpha i} \mathrm{H}^{\alpha i}\right)}
$$

and, according to our conventions about indexes,

$$
\Delta^{\alpha \lambda}=\left|y_{\rho \sigma}+y_{\rho} l x^{l \sigma}\right|_{\alpha \lambda}
$$

and

$$
\Delta^{\alpha i}=\left|y_{\rho \sigma}+y_{\rho l} x^{l \sigma}\right|_{\alpha \lambda} x^{i \lambda}
$$

7. The Legendre condition in the canonical variables. In order to abbreviate the notation, we contract the linear form

$$
B^{\alpha \lambda} s_{\alpha \lambda}+B^{\alpha i} s_{\alpha i}
$$

to

$$
B^{\alpha(\lambda, i)} S_{\alpha(\lambda, i)}
$$

and the quadratic form

$$
C^{\alpha \lambda, \beta \mu} s_{\alpha \lambda} s_{\beta \mu}+C^{\alpha \lambda, \beta j} s_{\alpha \lambda} s_{\beta j}+C^{\alpha i, \beta \mu} s_{\alpha i} s_{\beta \mu}+C^{\alpha i, \beta j} s_{\alpha i} s_{\beta j}
$$

to

$$
C^{\alpha(\lambda, i), \beta(\mu, j)} s_{\alpha(\lambda, i)} s_{\beta(\mu, j)} .
$$

With this convention, the Legendre condition in the canonical variables can be stated as follows: Under the side conditions

$$
\delta_{\alpha \beta} H^{\lambda(\mu, j)} s_{\beta(\mu, j)}=0,
$$

the quadratic form

$$
\lambda\left[\mathrm{H}^{\alpha(\lambda, i), \beta(\mu, j)}-\frac{m\left|\begin{array}{ll}
\mathrm{H}^{\alpha(\lambda, i)}, \mathrm{H}^{\alpha(\mu, j)} \\
\mathrm{H}^{\beta(\lambda, i)}, \mathrm{H}^{\beta(\mu, j)}
\end{array}\right|}{y_{\gamma(\nu, k)} \mathrm{H}^{\gamma(\nu, k)}}\right] s_{\alpha(\lambda, i) s_{\beta(\mu, j)}}
$$

must have positive characteristic numbers except for $p$ of them which are zero in consequence of the differential equations (2.2). The coefficient $\lambda$ in (7.6) is given by (6.5). 
8. The canonical differential equations and the Euler equations. An extremal which lies in a geodesic field satisfies the canonical differential equations

$$
\begin{gathered}
\mathrm{H}^{\alpha \kappa} \frac{\partial x^{i}}{\partial t^{\kappa}}=\mathrm{H}^{\alpha i}, \\
\mathrm{H}^{\beta \mu} \frac{\partial y_{\beta \alpha}}{\partial t^{\mu}}=-\mathrm{H}_{\alpha}, \\
\mathrm{H}^{\beta \mu} \frac{\partial y_{\beta i}}{\partial t^{\mu}}=-\mathrm{H}_{i}
\end{gathered}
$$

and the symmetry conditions

$$
\frac{\partial y_{\alpha \mu}}{\partial t^{\nu}}+\frac{\partial y_{\alpha i}}{\partial t^{\nu}} \frac{\partial x^{i}}{\partial t^{\mu}}=\frac{\partial y_{\alpha \nu}}{\partial t^{\mu}}+\frac{\partial y_{\alpha i}}{\partial t^{\mu}} \frac{\partial x^{i}}{\partial t^{\nu}} .
$$

These equations are sufficient to characterize an extremal. They imply the differential equations (2.2) which are the side conditions of the Lagrangian problem, and the Euler equations

$$
\frac{\partial\left(F_{i \alpha}+\mu^{\lambda^{\prime}} G_{\lambda^{\prime}, i \alpha}\right)}{\partial t^{\alpha}}=F_{i}+\mu^{\lambda^{\prime}} G_{\lambda^{\prime}, i} .
$$

9. Final remark. We also established a canonical theory of the parametric Lagrangian multiple integral problems with variable boundaries. So far as it is possible it resembles the above nonparametric theory. Both theories, however, are independent from each other and show some essentially different features.

\section{REFERENCES}

1. Constantin Carathéodory, Über die Variationsrechnung bei mehrfachen Integralen, Acta Szeged Sect. Sci. Math. 4 (1929), 193-216.

2. - Variationsrechnung und partielle Differentialgleichungen erster Ordnung, Teubner, Leipzig, 1935.

8151 Kreuzstrasse bei Holzkirchen, Germany 